\title{
Pionierarbeit mit medizinischer Abschlussprüfung?
}

\author{
Rudolf Probst, Alexander Huber
}

beide Prof. Dr. med., Klinik für Ohren-, Nasen-, Hals- und Gesichtschirurgie, UniversitätsSpital Zürich

Frau Guttormsen und Herr Perruchoud wiesen in ihrem Artikel in der SÄZ [1] auf die Pionierarbeit der Schweizerischen Medizinischen Fakultäten im Rahmen der neuen Eidgenössischen Abschlussprüfung (EP) nach dem Medizinstudium hin. Sie bezeichneten die Prüfung als zuverlässig und gerecht. Sie strichen sogar ihre Bedeutung für die Patientensicherheit hervor. Herr Fey wies in seinem Kommentar zum Artikel [2] auf Unklarheiten beim Bemessen des Erfolgs dieser neuen Prüfungsform hin - mindestens teilweise zu Recht, wie sich aus der Replik der Autoren ergab [3].

Prüfungen wie die EP oder das alte Staatsexamen, die übergeordnet von Lehrinstitutionen und somit unabhängig vom konkreten Lehrbetrieb durchgeführt werden, müssen primär als Instrument der Standardisierung und Reglementierung betrachtet werden. Sie bemessen, wie erfolgreich eine Lehrinstitution ihre Absolventen auf eine solche Prüfung und ihre Standards vorbereitet. Andere Qualitäten einer Ausbildung wie die Vorbereitung für die praktische Berufstätigkeit oder der Beitrag zur Patientensicherheit können damit nicht nachweislich verbessert werden. Es muss dahingestellt bleiben, ob die neue EP bessere Ärzte macht, oder auch ob das frühere Staats-

Un travail de pionnier avec l'examen fédéral de médecine humaine?

L'examen final en médecine humaine est tout d'abord un outil de standardisation et de réglementation et n'est pas en mesure d'apporter une amélioration substantielle de la qualité de la formation. L'examen final écrit est fortement standardisé depuis longtemps et I'on cherche désormais à faire de même pour l'examen pratique. La nouvelle formule élaborée ces dernières années est appropriée pour évaluer les compétences en communication et les examens simples sans résultats pathologiques, mais par rapport à l'ancien, ce nouvel examen pratique comporte d'importants inconvénients tels que l'impossibilité à évaluer l'aptitude à réaliser un examen de haut niveau, un ancrage insuffisant dans la pratique et un manque d'interaction entre examinateur et candidat. Il est impératif d'améliorer ce nouvel examen, car il vaut mieux standardiser le déroulement de l'examen que de standardiser le patient par un comédien. examen dies tat. Die Weiterbildung (WB) zum Facharzt in der Schweiz ist in diesem Zusammenhang illustrativ. Bis vor noch nicht allzu langer Zeit wurde die WB zum grossen Teil ohne Schlussprüfung durchgeführt. Nach unserer Erfahrung und unserem Dafürhalten hat die Einführung von Fachexamen nicht zu besseren Fachärzten beigetragen. Uns ist zumindest kein Beleg dafür bekannt und eine Krux liegt natürlich in der praktischen Unmöglichkeit der Definition und Erfassung eines «guten» Arztes.

\section{Neue praktische Prüfung}

Da das Staatsexamen seit mehr als 100 Jahren existiert, ist die Standardisierung und Reglementierung hier so selbstverständlich, dass sie nicht grundsätzlich hinterfragt wird. Standards, Reglemente und gesell-

\section{Die Prüfung findet in einem künstlichen} Rahmen ohne echte Patienten und oft ohne wirkliche Untersuchung statt.

schaftliche Normen wandeln sich teils erheblich in solch langen Zeitabschnitten und eine Anpassung der Prüfung ist normal. Die schriftliche Prüfung wurde mit dem MC in der Medizin schon vor geraumer Zeit hoch standardisiert und es bemühen sich ganze Institute um die ortsunabhängige Durchführung einer gleichförmigen ("gerechten») und in sich schlüssigen («reliablen») Prüfung. Nun hat dieser Trend auch die mündliche, "praktische» Prüfung erfasst und auch hier kümmern sich ganze Institute darum.

Mit Herrn Fey zusammen bezweifeln wir, ob mit dem neuen Format wirklich ein Mehrwert erreicht und die adäquate Form gefunden wurde. Die vorherige Prüfung war wenig standardisiert und formalisiert, wodurch fast sicher mehr Ungleichheiten und Ungerechtigkeiten entstanden. Sie hatte aber den deutlichen Vorteil der Anpassungsfähigkeit mit der Möglichkeit von direkten Interaktionen an die klinischen und examinatorischen Umstände. Der Lehr- und Lerneffekt dieser Prüfung war wohl wesentlich grösser als im heutigen Format. Die Befunde und Problematik der Prüfungs- 
patienten sassen bei den Kandidaten tief und begleiteten sie für die weitere ärztliche Tätigkeit.

Das heutige Format hat den unbestrittenen Vorteil, dass die Prüfungen sorgfältig vorbereitet und hervorragend organisiert sind. Gemäss einer klinikinternen Evaluation bei den Examinatoren der ORL kann sich das Format zudem zur Testung von Kommunikations-Skills eignen. Auch einfache und hoch standardisierte Untersuchungen vom Typ Blutdruckmessung oder Stimmgabelprüfung können getestet werden, solange sie keine pathologischen Befunde voraussetzen.

Dem stehen wesentliche Nachteile wie die Unmöglichkeit einer Prüfung von Untersuchungs-Skills auf höherem Niveau, ein fehlender Realitätsbezug und eine fehlende Interaktion zwischen Examinator und Kandidatin gegenüber.

\section{Beurteilung der Untersuchungs-Skills}

Ein wesentlicher Teil der praktischen Prüfung ist die Beurteilung der Untersuchungs-Skills. Es ist aber für die Kandidaten nicht nötig und oft sogar nicht möglich oder erwünscht, Untersuchungs-Skills auf höherem Niveau zu zeigen. Oft ist nur eine Geste oder eine angedeutete Ersatzhandlung im Sinne «Machen als ob» verlangt und ganz zur Farce wird die Skills-Prüfung, wenn die Untersuchung nur benannt und nicht durchgeführt werden darf, wie das beispielsweise in der ORL im Fall der Rhinoskopie oder Laryngoskopie vorkam. Die Belastung einer solchen Untersuchung wäre für die Schauspieler tatsächlich nicht klein, womit aber das Spektrum der eingeschlossenen Untersuchungen wesentlich eingeschränkt wird. Auch das Verständnis und die klinisch entscheidenden Umstände einer

\section{Die neue praktische Prüfung ist insgesamt zu} statisch und zu oberflächlich.

Untersuchung wie der physiologische oder pathologische Hintergrund, die Anwendung und die Befunderhebung können nicht geprüft werden. Eine Standardisierung auf diesem Niveau muss für manche Untersuchung als wenig sinnvoll erachtet werden. Die Beurteilung der tatsächlichen Untersuchung und deren Qualität bleibt bei dieser Form der praktischen Prüfung weitgehend auf der Strecke.

\section{Fehlender Realitätsbezug}

Die Prüfung findet in einem künstlichen Rahmen ohne echte Patienten und oft ohne wirkliche Untersuchung statt. Die Kandidatinnen sind sich diesem fehlenden Realitätsbezug sehr bewusst und können mehr oder weniger gut damit umgehen. Das Resultat der Prüfung hängt bestimmt mit der Tatsache zusammen, ob sich ein Kandidat gut oder schlecht

Das tatsächliche Können und Wissen wird noch ungenügender als früher geprüft.

auf diese künstliche Situation einstellen kann und weniger mit seinen tatsächlichen klinischen Fähigkeiten.

Schauspieler können selbstverständlich pathologische Befunde wie eine vergrösserte Leber, ein gerötetes Trommelfell oder ein Ulcus der Schleimhaut nicht simulieren, was unvermeidlich ein weiterer wesentlicher Mangel des Realitätsbezugs darstellt. Durch diesen Umstand alleine ist das Spektrum der heutigen Prüfungsform vermutlich bereits mehr eingeschränkt als bei der vormaligen Prüfung mit Patienten. Zudem fallen Kinder und andere heikle Patientengruppen zum Vornherein weg.

Hybridformen, in denen Befunde mit einem Bild oder Modell ersetzt werden, sind keine Lösung. Sie verhindern das im Alltag entscheidende «Finden» des Befundes und führen zum oben bereits erwähnten oberflächlichen Gestenverhalten. Unsere Erfahrungen mit Modellen in der ORL sind zudem sehr schlecht und wir kennen persönlich keine geeigneten Modelle für die Befunderhebung in der ORL.

\section{Interaktion Kandidatin - Examinatorin}

Die Prüfqualität sowie die Motivation der Kandidaten und Examinatoren leiden unter der fehlenden Interaktion, die ausdrücklich untersagt ist. Eine Blockade wegen Nervosität, ein Missverständnis oder ein Versprecher können grundsätzlich nicht korrigiert werden. In der ORL wurde die Kommunikation des Examinators teils auf die Mitteilung beschränkt, dass die Kandidaten keine Rhinoskopie oder Laryngoskopie durchführen sollen, was bei einem Teil der Kandidaten eine Verunsicherung verursachte. Sie bekamen den Eindruck, auf der falschen Spur zu sein oder die Situation falsch beurteilt zu haben und sie gerieten dadurch gelegentlich in ihrer Untersuchungsabfolge aus dem Rhythmus.

Die untersagte Interaktion erlaubt es auch nicht, das Wissen um Zusammenhänge oder ein Grundverständnis zu den Untersuchungen und zur Klinik zu prüfen.

\section{Weiterentwicklung nötig}

Die neue praktische Prüfung ist insgesamt zu statisch und zu oberflächlich. Das tatsächliche Können und 
Wissen wird noch ungenügender als früher geprüft und das klinische Spektrum ist vermutlich begrenzter als in der alten Form. Die Prüfung ist zudem für Kandidaten und Examinatoren auf mehreren Ebenen motivationshemmend. Dazu tragen die erwähnte fehlende Kommunikation und vermutlich auch die hohe Standardisierung bei. Diese schränkt das mögliche Prüfungsspektrum zu sehr ein und damit auch das Lernspektrum. Jede Prüfung stellt leider unvermeidlich ein Hauptmotivator für das Lernen dar und gerade deshalb ist ein offenes Prüfungsformat mit dem möglichen Einbezug einer breiten Klinik und aller Skills wichtiger als die hohe Standardisierung im Namen der Gerechtigkeit.

\section{Hybridformen, in denen Befunde mit einem Bild oder Modell ersetzt werden, sind keine Lösung.}

Das ideale Format der Prüfung ist nicht gefunden, es soll und kann verbessert werden. Das Gesetz gibt keine Vorgaben zur gleichzeitigen, uniformierten und ortsunabhängigen Durchführung, es verlangt lediglich eine eidgenössischen Prüfung, die «abklärt, ob die Studierenden über die fachlichen Kenntnisse, Fertigkeiten und Fähigkeiten sowie über die Verhaltensweisen und die soziale Kompetenz verfügen, die sie zur Ausübung des entsprechenden Medizinalberufes benötigen; und die Voraussetzungen für die erforderliche Weiterbildung erfüllen» (Art. 14-2, MedBG).

Diese Vorgabe erlaubt eine offenere Gestaltung und kann durchaus Prüfungen mit Patienten einschliessen. Solche Prüfungen sind wohl tatsächlich kaum an einem Tag in der ganzen Schweiz zu organisieren, was aber nicht nötig erscheint und machbar ist, wie wir versuchsweise in der ORL in Zürich gezeigt haben. Wir führten für den gesamten 6. Jahreskurs ein MiniCEX als Abschluss unseres klinischen Kurses durch. In einer vorgegebenen Prüfungszeit wurden von den
Kandidaten Patienten untersucht, die möglichst ein häufiges Krankheitsbild mit Relevanz für die allgemeine Medizin wie Cerumen, Rhinitis, Pharyngitis oder Schwerhörigkeit aufwiesen. Es wurde eine gezielte Anamnese, eine gezielte Untersuchung mit Befunderhebung, -beschreibung und -demonstration sowie Therapievorschläge erwartet. Der Examinator hatte die Aufgabe, den Examensablauf mit Hilfe von Protokollen und Checklisten zu standardisieren, die dem Themenkreis zugeordnet waren. Er hatte das Recht und die Pflicht, das Examen entlang dieser vorgegebenen Linien zu gestalten und die Checkpunkte nachzufragen, die auch das Verständnis von Zusammenhängen enthielten. Fragen zum Verständnis des Krankheitsbildes oder der Untersuchung, zu differentialdiagnostischen Überlegungen sowie zu wichtigen Prognosefaktoren wie gefährliche Komplikationen waren ebenfalls enthalten. Die Bewertung dieses Examens durch die Kandidaten und Examinatorinnen war sehr gut.

Diese praktische Examensform versuchte vielmehr die Examinatoren und den Ablauf des Examens zu standardisieren als die Patienten und die klinische Situation. Die heutige praktische Prüfung versucht beides, wodurch der Realitätsbezug leidet und die Standardisierung der Examinatoren ungeeignet erfolgt, in dem die Kommunikation zwischen Examinator und Kandidat unterbunden wird. Wir glauben, dass gerade für chirurgische Fächer und die Pädiatrie Formen mit echten Patienten und klinischen Situationen gefunden werden müssen, was die Verwendung von Schauspieler für geeignete Situationen nicht ausschliessen soll.

Literatur

1 Guttormsen S, Perruchoud AP. Pionierarbeit für die Abschlussprüfung Medizin. Schweiz Ärztezeitung. 2015;96(50-51):1846-8.

2 Fey M. Annuntio vobis gaudium magnum - ein neues klinisches Staatsexamen! Schweiz Ärztezeitung. 2016;97(4):153-4.

3 Guttormsen S, Perruchoud AP. Die neue eidgenössische Prüfung hat einen Mehrwert. Schweiz Ärztezeitung. 2016;97(4):155-6. 